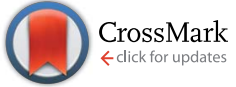

Cite this: RSC Adv., 2015, 5, 79270

Received 9th July 2015

Accepted 11th September 2015

DOI: $10.1039 / c 5 r a 13419 f$

www.rsc.org/advances

\section{Encapsulation of food protein hydrolysates and peptides: a review}

\author{
Aishwarya Mohan, ${ }^{a}$ Subin R. C. K. Rajendran, ${ }^{a}$ Quan Sophia He, ${ }^{b}$ Laurent Bazinet ${ }^{c}$ \\ and Chibuike C. Udenigwe*a
}

Food protein hydrolysates and peptides are considered a category of promising functional food ingredients. However, commercial application of protein hydrolysates and their constituent peptides can be impeded by their low bioavailability, bitter taste, hygroscopicity and likelihood of interacting with the food matrix. Encapsulation as a delivery mechanism can be used to overcome these challenges for improving the bioavailability and organoleptic properties of the peptides. Proteins, polysaccharides and lipids are the three carrier systems that have been utilized in food peptide encapsulation. The protein and polysaccharide systems mainly aim at masking the bitter taste and reducing the hygroscopicity of protein hydrolysates, whereas the lipid-based systems are intended for use in enhancing the bioavailability and biostability of encapsulated peptides. A spray drying technique is largely used to achieve microencapsulation in both protein and polysaccharide systems while, generally, liposomes are prepared by a film hydration technique. However, it is seen that the encapsulation efficiency (EE) of peptides using the liposome model is relatively lower since the entropy-driven liposome formation is uncontrolled and spontaneous. Achieving adequate EE through cost effective techniques is indispensable for encapsulation to be applicable to bioactive peptide-based product commercialization. Furthermore, the design of high quality functional foods requires detailed understanding of the release mechanism and kinetics, gastrointestinal stability, bioavailability and physiological bioactivity of the encapsulated peptide products.

\section{Introduction}

Encapsulation is the process of packaging solid, liquid or gaseous materials in capsules of different carriers, which release active compounds (by diffusion, dissolution, $\mathrm{pH}$ trigger, degradation, etc.) at various intervals depending on the stability of the capsule. ${ }^{1}$ The pharmaceutical industry has extensively used encapsulation technology in drug delivery to achieve precise, controlled, stable and targeted delivery of the drug. The food industry has also embraced the process of encapsulation to overcome certain challenges arising as a result of growing demand for functional ingredients in food. ${ }^{\mathbf{1}}$ This review is focused on bioactive food protein hydrolysates and peptides, whose incorporation into functional foods can be hindered by several challenges such as bitter taste, hygroscopicity, hydrophobicity, reaction with the food matrix, incompatibility, limited bioavailability, and biostability. ${ }^{2}$ Biostability and bioavailability are pivotal for achieving physiological benefits as

${ }^{a}$ Food Functionality and Health Research Laboratory, Department of Environmental Sciences, Faculty of Agriculture, Dalhousie University, Truro, NS, B2N 5E3, Canada. E-mail: cudenigwe@dal.ca; Fax: +1-902-843-1404; Tel: +1-902-843-6625

${ }^{b}$ Department of Engineering, Faculty of Agriculture, Dalhousie University, Truro, NS, B2N 5E3, Canada

${ }^{c}$ Department of Food Science and Nutrition, Institute of Nutrition and Functional Foods, Université Laval, Québec, QC, G1V 0A6, Canada the peptides need to reach their targets intact in order to exert their bioactivity. Encapsulation has been used in the food industry and for delivery of several bioactive compounds that are sensitive to environmental factors, such as polyphenols, carotenoids and omega-fatty acids. ${ }^{3}$ Nevertheless, encapsulation is yet to be applied in the commercial production of bioactive food protein hydrolysates and peptides.

Bioactive peptides are different from other food bioactive compounds such as vitamins or polyphenols in that the chemical species within the protein hydrolysates are highly heterogeneous. ${ }^{4}$ Consequently, bioactive peptides may need to be isolated from more complex matrices or fractionated prior to encapsulation. Most studies on bioactive peptides are focused on the discovery of new bioactivity and protein precursors and elucidation of mechanisms with limited attention given to their biostability and bioavailability. Encapsulation can be explored for the delivery of bioactive food peptides; however, it is seen that optimum conditions for encapsulation of other compounds do not necessarily apply to bioactive peptides. Currently, there is a dearth of literature expounding various aspects of encapsulation in relation to food protein-derived bioactive peptides. Bioactive peptides are primarily encapsulated for the purpose of masking the bitter taste that result from exposure of taste receptors to hydrophobic amino acid residues generated from protein hydrolysis. ${ }^{5}$ Another major objective of 
encapsulation is the reduction of hygroscopicity to ensure textural and storage stability of protein hydrolysates and peptides. Bioavailability and stability of the peptides are rarely investigated as major concerns despite strong evidence indicating that in vitro bioactivity are not always replicated in animal models and human subjects. The roles of several factors related to the process of encapsulation of food protein hydrolysates and peptides including the type of carrier system, method used for encapsulation, purity of wall/carrier material, core-to-wall ratio, and encapsulation conditions are still not clearly understood. This review highlights current advances in the process of encapsulation for food protein hydrolysates and peptides including factors that determine encapsulation efficiency (EE), and knowledge gaps that exist in the use of encapsulation for achieving the highest possible potential for food-derived bioactive peptides.

\section{Need for peptide encapsulation}

A primary challenge faced in translating food protein-derived bioactive peptides into commercial products is the susceptibility of peptides to gastrointestinal (GIT) digestion with the risk of losing their structural integrity and function when hydrolysed by GIT proteases and peptidases. ${ }^{2,6}$ Bioavailability is used to depict the portion of the bioactive compound that is unchanged, absorbed and that reaches the systemic circulation. ${ }^{3}$ Bioactive peptides, when orally administered, are subjected to peptic digestion in the stomach under acidic conditions, $^{7}$ followed by several alkaline pancreatic protease digestion in the intestinal phase before being absorbed through the enterocytes. It has been understood that oral ingestion of bioactive peptides will expose them to the action of at least 40 different enzymes before reaching systemic circulation. ${ }^{7}$ Several studies have demonstrated that most food protein-derived bioactive peptides containing more than 2-3 amino acid residues do not withstand simulated gastrointestinal enzymatic digestion. ${ }^{7}$ However, the bioactivity of some peptides have been retained or even increased following simulated GIT proteolytic activities. Particularly, dairy-derived antihypertensive tripeptides Val-Pro-Pro and Ile-Pro-Pro, already commercially available for consumption through functional foods, are among the very few peptides that are reported to be stable following GIT digestion. Protecting bioactive peptides from physiological modifications is essential in translating in vitro activities in animal models and humans. Therefore, encapsulation has become a relevant and important technology for enhancing the utilization of food-derived bioactive peptides for human health promotion.

\section{Type of carrier systems for peptide encapsulation}

The food industry is restricted to the use of carrier matrices that are edible, biodegradable, non-toxic and inexpensive. ${ }^{3}$ Although there are separate extensive reviews on lipids, ${ }^{8}$ polysaccharides ${ }^{3}$ and protein-based ${ }^{9}$ carriers for encapsulation of food-derived bioactive compounds, there is a need to discuss the different carriers with particular focus on their use for encapsulating food protein hydrolysates and peptides (Fig. 1).

\subsection{Protein-based carriers}

Encapsulation using the protein-based matrix is thought to be the most nutritionally beneficial system. ${ }^{10}$ Despite the popularity of protein-based carriers for delivering other food bioactive compounds such as flavonoids, vitamins and $\beta$-carotene, ${ }^{9}$ the use of protein carriers in bioactive peptide encapsulation is limited. Encapsulating bioactive core substance with a chemically similar material is challenging because of structural similarity; that is, the encapsulation shell is predicted to face instability issues similar to the encapsulated bioactive compound. ${ }^{5}$ Recently, Wang et al. reported the use of native, acylated and high pressure-treated rapeseed protein isolate for the encapsulation of peptides derived from the same material. ${ }^{11}$ The inclination towards the use of proteins for delivery of bioactive compounds is due their functional properties such as film and gel forming ability, emulsification and solubility, in

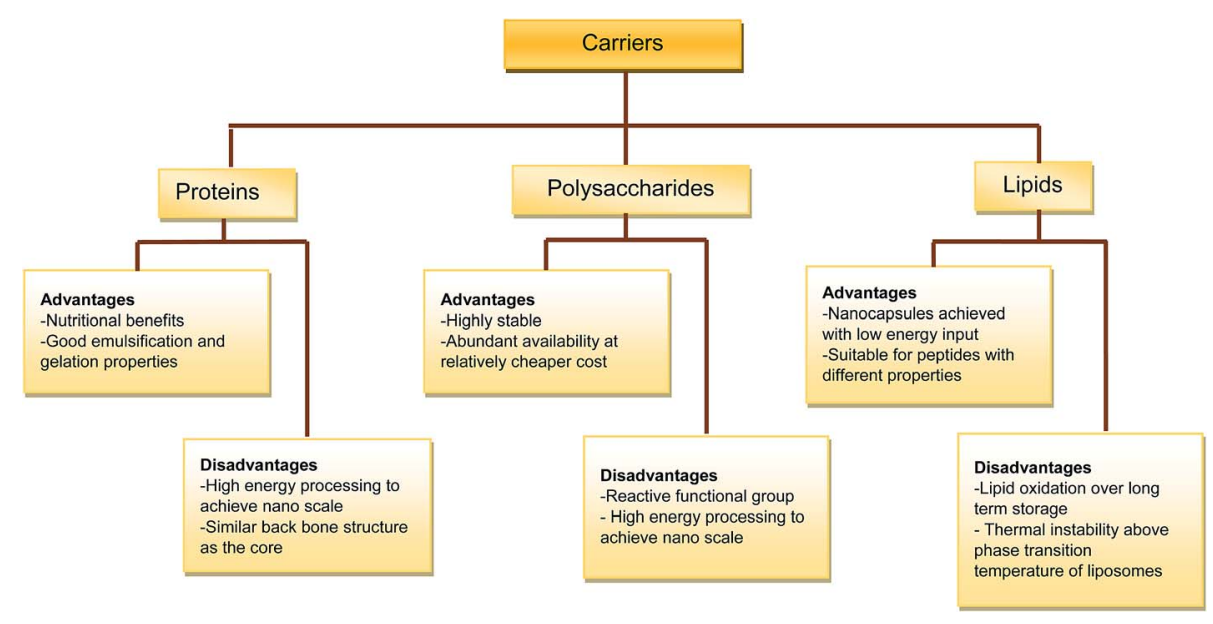

Fig. 1 Carriers used for encapsulation of protein hydrolysates and peptides. 
addition to their nutritional benefit as sources of essential amino acids. Among the protein sources, soybean has been the predominant choice for bioactive peptide encapsulation (Table 1) whereas milk proteins are extensively used in the encapsulation of other non-peptide bioactives. ${ }^{9}$ Milk caseins have been used for encapsulation of small hydrophobic compounds due their micellar structure in aqueous environment. ${ }^{12}$ However, it appears that there is no clear rationale for selection of the protein carrier for food protein hydrolysate and peptide encapsulation. The encapsulation mechanism involving hydrophilic or hydrophobic interactions appear challenging to achieve with protein carriers considering the structural heterogeneity of the encapsulated peptide mixtures. Moreover, recent studies have reported successful encapsulation of dipeptide Phe-Trp and pentapeptide Leu-Trp-Met-ArgPhe using $\mathrm{CaCl}_{2}$ cross-linked whey protein microbeads of 1-2 mm diameter, resulting in equilibrium constants of 2.3 and 37 , respectively for the peptides. ${ }^{13,14}$ This demonstrates that the peptides are more distributed in the protein microbeads compared to the aqueous phase, with higher distribution and EE observed for the pentapeptide. Although not extensively used as carriers for peptide encapsulation, milk proteins are well established as major sources of bioactive peptides. Furthermore, polysaccharides can be combined with the protein carriers to provide structural stability to the encapsulation (Table 1). Although a "top-down" approach, involving fragmentation of larger structures, has been proposed to accomplish nanoencapsulation, ${ }^{9}$ only microencapsulation has been achieved to date when proteins are used for peptide encapsulation. Protein carriers have been shown to reduce the hygroscopicity of peptides, ${ }^{10,15}$ although there are contrasting reports of increased hygroscopicity after encapsulation. ${ }^{5}$ This variation could be attributed to physical and structural changes that can occur with the processing of proteins during encapsulation.

\subsection{Polysaccharide-based carriers}

Polysaccharides are generally ideal for use as delivery agents because they are structurally stable, abundant in nature and inexpensive. The reactive functional groups of polysaccharides make them one of the best choices as carrier matrices. ${ }^{3}$ On the other hand, under extreme conditions, such as high temperature, the polysaccharide wall is susceptible to reacting with the peptide core to form complex products (e.g. Maillard reaction products), which can be potentially toxic and also deplete the bioactive peptides. In order to circumvent this challenge, the reactive functional groups of polysaccharides have been modified by processes such as carboxymethylation to produce relatively inert carriers. ${ }^{16}$ The colossal molecular structure of polysaccharides contributes to their stability as carriers during production and processing of encapsulated products. Polysaccharides derived from plants, animals and microbial sources, such as gum arabic, chitosan, cyclodextrin and maltodextrin, have been utilised for food protein and peptide encapsulation (Table 1). Although polysaccharides are mostly used in combination with protein carriers, Yokota et al. used disaccharides as cryoprotectants in the liposome encapsulation model. ${ }^{17}$ In the study, addition of disaccharides was found to reduce the EE and increase the particle size of the products. Furthermore, the amount of polysaccharide carriers used was found to positively correlate with particle size of the encapsulated products. ${ }^{18}$

\subsection{Lipid-based carriers}

Liposphere and liposome are two lipid-based systems that are currently used for encapsulating food protein hydrolysates and peptides. The former has a fatty acid inner layer and outer layer composed of the hydrophilic part of the fatty acid or phospholipid (PL), whereas the latter is a single or multiple concentric bilayer made of phospholipids constituting a vesicle. ${ }^{4}$ Accordingly, lipospheres appear appropriate for encapsulating hydrophobic peptides that can interact with the hydrophobic inner layer of the carrier. A few studies have used lipospheres for the encapsulation of protein hydrolysates with moderate to high EE. For instance, a combination of stearic acid and phosphatidyl choline (PC) was used to encapsulate casein peptide fractions by the melt process, and this resulted in different (50-83\%) EE, even when the samples possess similar surface hydrophobicity. ${ }^{19}$ This suggest that the EE of peptides in lipospheres can be affected by other factors. Similar EE (74\%) was also reported for $\mathrm{CH}$ encapsulation in multi-component lipid carrier (stearic acid/cupuacu butter/polysorbate 80). ${ }^{20}$ Peptide encapsulation was found to not affect the thermal behaviour of the capsules ${ }^{20}$ and no considerable oxidation was observed during a 60 day storage of the encapsulated products. ${ }^{19}$ The latter can be attributed to the predominant composition of saturated stearic acid and absence or small amounts of oxidatively-labile unsaturated fatty acids in the spheres.

Liposome is a more popular encapsulation carrier compared to the liposphere, which would be less preferred for food applications because of its high saturated fatty acid content, and the limited choice of substances that can be incorporated in its highly hydrophobic core. However, liposome is compatible with a wide variety of bioactive peptides. The aqueous core appears suitable for hydrophilic peptides and other compounds, while the interior of the bilayer is compatible with hydrophobic peptides. Moreover, amphiphilic peptides can exist at the interface between the shell and core of the liposome structure, which would interact with the hydrophobic and hydrophilic amino acid residues, respectively. ${ }^{17}$ Liposome is similar to cell membranes and is therefore favourable for the delivery of bioactive compounds, which can otherwise be degraded by the digestive physiological environment. PC is the commonly used phospholipid for liposome preparation. The large, commercial-scale production that is possible in the case of lipid carriers is a distinct advantage of liposomes over other carrier systems. ${ }^{8}$ Liposomes adapted from the pharmaceutical industry have certain shortcomings in functional food application. Particularly, the thermal instability of liposome encapsulated food peptide products beyond the phase transition temperature of the phospholipid can limit their incorporation in thermally processed food. Besides, liposome preparation 


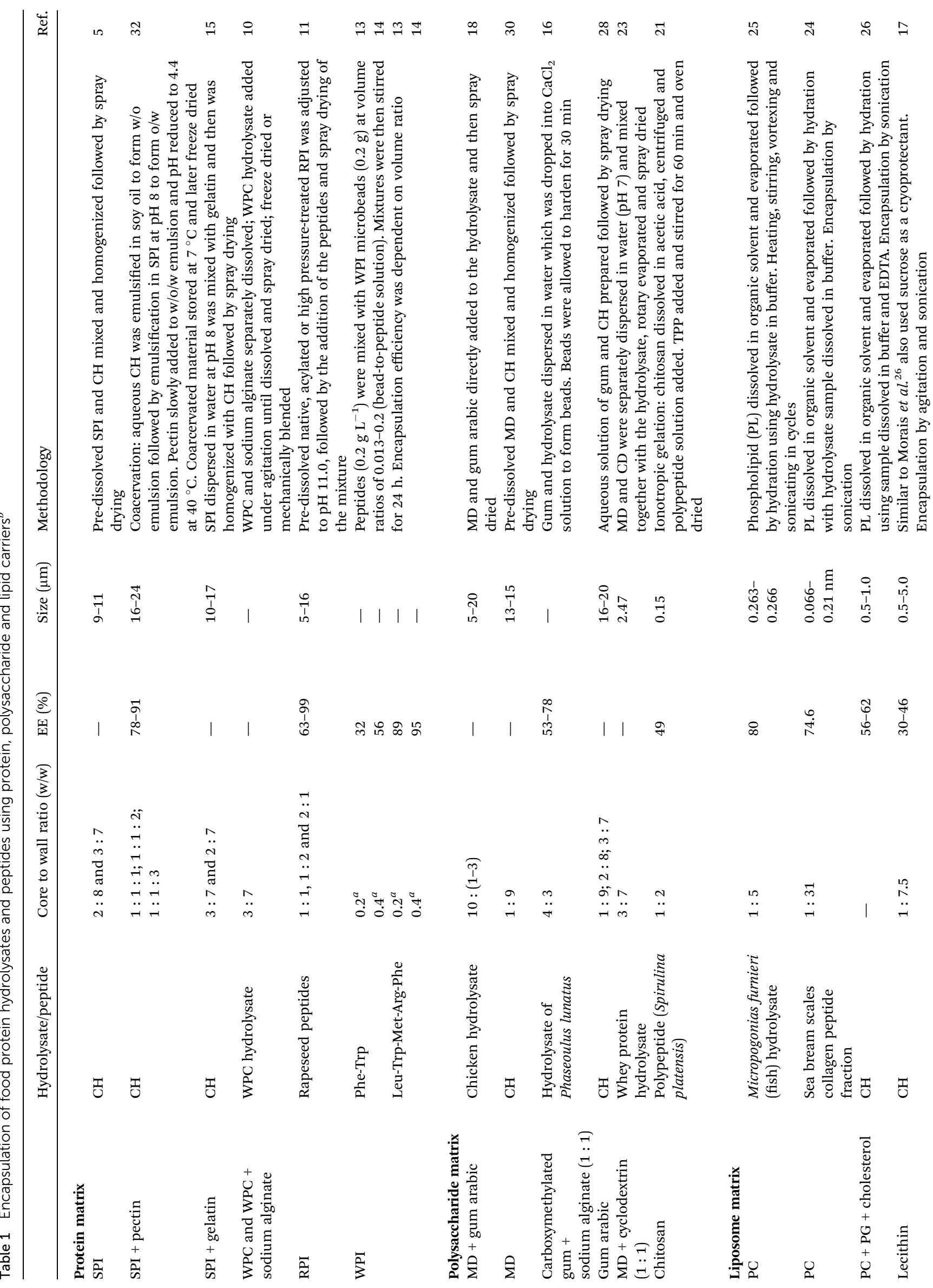


involves the use of cholesterol to increase the stability of the lipid bilayer, which is a health concern for application in functional foods. Yet another drawback of using liposome system in peptide encapsulation is the risk of lipid oxidation during production, processing and storage of the products. Consequently, the presence of lipids (especially unsaturated fatty acids) in the peptide-based functional foods can impact product shelf life and limit the choice of processing and storage conditions. Mild oxidation was reported for liposomes at high temperature and low $\mathrm{pH},{ }^{21}$ although this needs to be reassessed when food protein hydrolysates and peptides are loaded in the capsules. Taken together, optimum conditions need to be developed to take advantage of the lipid-based system in food protein hydrolysate and peptide encapsulation considering the health and product quality challenges posed by the use of saturated and unsaturated lipids in lipospheres and liposomes, respectively.

\section{Criteria for determining the quality of peptide encapsulation}

\subsection{Particle size}

The dispersibility and solubility of the encapsulated peptide product greatly depend on the particle size. Particle size of above $50 \mu \mathrm{m}$ can significantly affect the solubility, dispersion and hence, the texture and feel of the food. ${ }^{15}$ Encapsulation products can be either of micro or nano scale. Nanoencapsulation is advantageous because of its high surface area that can increase the solubility and bioavailability of the product. It is thought that the smaller size of the capsules enhances delivery or release of the active molecules. ${ }^{8}$ Among the various carriers, the lipid-based systems are more efficient for preparing nanoencapsulated protein hydrolysate and peptide products compared to the protein or polysaccharide systems. Due to their large molecular structure, most encapsulation involving protein and polysaccharide carriers, or a combination of both, results in the production of microcapsules. The combination of proteins and polysaccharides in encapsulation generates relatively large capsules, although all peptide encapsulation studies to date have yielded products with particle sizes under the threshold value of $50 \mu \mathrm{m}$ (Table 1). However, Zhang et al. have produced $150 \mathrm{~nm}$ nanoencapsulated spirulina protein hydrolysates using chitosan as carrier. ${ }^{22}$ Apart from the type of carrier, the particle size of the encapsulated peptide products also depends on the method used for encapsulation. ${ }^{9}$ Yang et al., in spite of using maltodextrin and cyclodextrin, were able to produce encapsulated products loaded with whey protein hydrolysates with particle sizes as small as $2.4 \mu \mathrm{m}$ using the spray drying method. ${ }^{23}$ Since spray drying is a destructive method of preparation, it is possible that the smaller particle size resulted from fragmentation of the capsules. Furthermore, the particle size of encapsulated peptides was found to depend on the core-to-wall ratio ${ }^{18}$ (see Section 5.3), but some studies have reported the absence of a particular trend. ${ }^{15,24}$ 


\subsection{Zeta potential}

Surface charge is one of the properties that convey the stability of encapsulated products. Stability enables the prediction of the behaviour of the encapsulated product in a food matrix. However, encapsulation performed for the purpose of masking the bitter taste of protein hydrolysates and peptides has not been focused on this surface property. Liposome-based encapsulation studies report high net negative zeta potential (surface charge) due to the presence of phospholipids, which have negatively charged hydrophilic heads. A decrease in the magnitude of the zeta potential would decrease the stability of the encapsulated product. Encapsulated protein hydrolysate and peptide products of low magnitude zeta potential have the tendency to aggregate in aqueous environment; a surface charge of $\pm 30 \mathrm{mV}$ is essential to form stable dispersion due to electrostatic repulsion of the particles. ${ }^{25}$ Encapsulation of peptides using chitosan yielded a product with a high positive surface charge of $+41.5 \mathrm{mV} .^{22}$ Although there is limited knowledge on surface charge dynamics of encapsulated food protein hydrolysates and peptides, Mosquera et al. reported that simultaneously increasing the concentration of components of both the core (sea bream scale collagen peptide fraction) and the wall (PC) reduces zeta potential. ${ }^{24}$ Most studies with polysaccharide and protein carriers did not report the zeta potential of the encapsulated protein hydrolysates and peptides. This information is particularly useful in evaluating the effects of the processing techniques utilized for these carriers, such as spray drying, on the encapsulated product stability. As discussed in Section 5.4, mild processing techniques such as film hydration and ionotropic gelation have so far resulted in stable encapsulated products. ${ }^{22,24}$

\subsection{Encapsulation efficiency}

EE can be defined as the amount of bioactive compound (peptide) trapped in the core or surface of the carrier compared to the initial amount of the bioactive material. Zavareze et al. measured EE of peptides indirectly by removing unencapsulated portion of the protein hydrolysate by centrifuging followed by estimation of peptide concentration using Lowry assay. ${ }^{25}$ Membrane ultrafiltration has also been used to separate unencapsulated hydrolysate from the capsules prior to protein quantification. ${ }^{15}$ Moreover, Morais et al. assessed the encapsulation rate of peptides in liposomes and lipospheres indirectly using second derivative spectrophotometry. ${ }^{26} \mathrm{EE}$ is an important factor to consider especially in producing commercial bioactive protein hydrolysate and peptide products. Although it was suggested that EE of over $50 \%$ increases the risk of leakage, ${ }^{8}$ lower EE would lead to inefficient use of the bioactive materials and also imply that higher amount of encapsulated products would be required to attain the peptide quantities needed to exert physiological bioactivities. EE depends on the core-to-wall ratio, the conditions in which encapsulation is carried out, and encapsulation technique or production method utilized. ${ }^{9}$ EE of microcapsules of protein hydrolysates and peptides prepared with polysaccharide carriers are occasionally reported (Table 1). Moreover, encapsulation using protein and polysaccharide carriers have resulted in higher EE compared to lipid-based (particularly liposome) peptide encapsulation (Table 1), possibly since the former is controlled and involves high energy processes in entrapping or encapsulating the peptides. Liposome formation involves entropy-driven, spontaneous and less controlled process. In general, techniques using high shear forces, pressure and high temperature result in higher EE, while mild preparation techniques such as film hydration and ionotropic gelation result in lower EE.

\section{Factors that can affect encapsulation of peptides}

The chemistry of the encapsulated bioactive material fundamentally affects the EE. Although, there is limited comprehensive knowledge about the impact of peptide structure on encapsulation, EE is also thought to partly depend on some other factors as discussed below.

\subsection{Peptide charge}

Encapsulation of casein-derived peptides using liposomes mostly resulted in low EE (14\%), which is attributable to the phosphoserine residues in caseinophosphopeptides (Mohan \& Udenigwe, unpublished data). PL and the phosphopeptides are highly negatively charged leading to molecular repulsion and reduced encapsulation. Similarly, liposomal encapsulation of negatively charged intact bovine serum albumin has also been reported to result in low EE of 34\%. ${ }^{27}$ Higher EE values have been reported for the encapsulation of protein hydrolysates from other sources that lack the phosphorserine residues, ${ }^{24,25}$ although the surface charge of the core materials was not reported.

\subsection{Type and purity of carrier/wall material}

Type and purity of carrier material are important factors that determine EE. High EE of $74-80 \%$ have been achieved using purified PC to form the liposomal carrier. ${ }^{24,25}$ The small difference in EE can be due to the nature and different sources of the protein hydrolysates and peptides used for encapsulation. However, encapsulation of a similar protein hydrolysate with crude soy lecithin resulted in low EE of $46 \%{ }^{15}$ Similarly, in liposphere-based encapsulation studies, EE was no less than $50 \%$ and a maximum of $83 \%$ EE was obtained using purified PC and stearic acid. ${ }^{\mathbf{1 9 2 6 , 2 8}}$ Conversely, comparable EE have been obtained when crude cupuacu butter was used with stearic acid in encapsulating casein peptide fractions. ${ }^{20}$ The high EE of liposphere encapsulation can generally be attributed to the affinity of hydrophobic peptides in the core to the hydrophobic stearic acid inner layer (in contact with the core), although this mechanism entails the exclusion of hydrophilic (possibly bioactive) peptides from the capsule. Apart from casein peptides, there is a dearth of information on encapsulation of other protein hydrolysates and peptides using lipospheres making it challenging to draw conclusions on the prospects of the carrier system. Hydrophobic interaction has also been reported to increase $\mathrm{EE}$ for peptide encapsulation using microbeads prepared from whey protein isolate as the carrier; 
the study found a linear relationship between hydrophobic column capacity factor ( $k$, depicting molecular hydrophobicity) and equilibrium constant $(K) \cdot{ }^{13}$ However, the study did not consider the role of peptide charge, which can also affect EE. Furthermore, acylation (by 47\%) and high pressure treatment (200-400 MPa) of rapeseed protein isolate carrier resulted in high amount of secondary structure ( $\alpha$-helix and $\beta$-sheet) and increased Young's modulus, which led to higher EE compared to the native and $5 \%$ hydrolyzed protein carriers. ${ }^{11}$

The advantage of using purified carrier materials is the reduction in the amount of materials needed to achieve high EE. Most polysaccharide-based encapsulation uses purified or synthetic carrier materials. Despite the consistently high EE, the use of high-purity wall materials in protein hydrolysate and peptide encapsulation does not appear to be economically feasible for the functional food industry, ${ }^{9}$ except perhaps for the protein carriers. Obtaining or purifying the wall material would add significant step to the production process and can increase the input and product unit costs. There is a need for further research to identify and adapt processes that will lead to adequate EE for bioactive peptides without requiring high-cost input.

\subsection{Core-to-wall ratio}

Typically, encapsulation involves the use of large amounts of wall materials than the active core compounds. EE is largely influenced by the core-to-wall ratio and is found to always decrease with increase in the core concentration, ${ }^{22,24}$ which can be due to overloading of the encapsulation system. Increase in the concentration of the wall material initially leads to increase in the EE until a certain point. For instance, Mosquera et al. reported a maximum $\mathrm{EE}$ of $74.6 \%$ at $1: 31.5(\mathrm{w} / \mathrm{w})$ core-to-wall ratio (PC and sea bream scale protein hydrolysate) ${ }^{24}$ the EE was found to decrease to $67 \%$ when the ratio was slightly changed to $1: 38.5(\mathrm{w} / \mathrm{w})$. Interestingly, Zavareze et al. achieved $80 \%$ EE of fish protein hydrolysate using a much lower core-towall (PC) ratio of $1: 5(\mathrm{w} / \mathrm{w}),{ }^{25}$ which suggests possible dependence of EE on the nature and molecular composition of the encapsulated material. However, Subtil et al. found that increase or decrease of the amounts of the wall (gum arabic) or core materials (casein hydrolysate) did not affect other characteristics such as the capsule morphology. ${ }^{29}$ In contrast, a few studies involving protein and polysaccharide carriers have reported that varying the core-to-wall ratio increases the mean particle size and alters the morphology of the encapsulated protein hydrolysate and peptide products. ${ }^{11,18}$ However, there has been no observable link between core-to-wall ratio and particle size for liposome-based encapsulated protein hydrolysates and peptides. ${ }^{24}$ The lack of relationship between liposomal size and concentration of the core or wall can be partly attributed to difference in the process used in encapsulation. Furthermore, core-to-wall ratio increase from $1: 2$ to $2: 1$ was found to increase the mean particle size and decrease the spray dry yield of peptide products encapsulated with rapeseed proteins. ${ }^{11}$ Volume ratio of the core and wall material is also important in determining EE. A recent study reported that high
EE (up to 95\%) can be achieved by increasing the volume ratio of whey protein microbeads and peptides in aqueous solution ( $V_{\text {bead }} / V_{\text {aq }}$ ) from 0.013 to $0.2 .^{13}$ To date, commonly used core-towall ratios are $3: 7$ and $2: 8$ for protein and polysaccharide carriers and $1:(5-10)$ for liposome carriers (Table 1).

\subsection{Techniques used for encapsulation}

Currently, several techniques have been proposed for use in encapsulation of bioactive compounds such as coacervation, spray cooling, extrusion, supercritical fluid extraction, cocrystallization and inclusion. ${ }^{9}$ Techniques involved in the encapsulation of food ingredients utilizing lipid, protein and polysaccharide-based carrier systems have been extensively reviewed., ${ }^{3,8,9}$ This review focuses only on the techniques currently applied and are relevant for encapsulation of food protein hydrolysates and peptides.

5.4.1. Film hydration. Liposome-based encapsulation of food protein hydrolysates and peptides mostly employs the film hydration technique. This option is popular due to the simple, yet effective mechanism where phospholipids self-assemble in response to energy input in the form of heat, agitation and sonication thereby trapping the aqueous core containing the peptides. The bilayer formation in liposome does not require the use of any sophisticated equipment except for the application of energy to drive the self-assembly. The disadvantage of liposome formation is that the uncontrolled assembly mechanism can lead to poor reproducibility and varying EE (Table 1). Moreover, organic solvents used in the liposomal encapsulation process need to be removed prior to use of the encapsulated products in functional foods, which introduces additional steps that can affect EE and the quality of the encapsulated products.

5.4.2. Spray drying. Both the protein and polysaccharidebased encapsulation frequently employ spray drying to achieve encapsulation due to the relatively low processing cost and ease of the technique. ${ }^{15}$ This technique involves forming droplets and spraying at high temperature resulting in dried particles. ${ }^{18}$ Unlike the liposome system, the spray drying process achieves entrapment of bioactives in the protein and polysaccharide carriers rather than having distinct wall and core. ${ }^{15}$ Spray drying has been found to result in microspheres with the active material uniformly distributed in the carrier, which typically occurs when the carrier and core materials are similarly hydrophilic. ${ }^{15}$ This phenomenon is expected to lead to high EE although no study has yet reported the EE of spray dried encapsulated protein hydrolysate and peptide microcapsules (Table 1). Moreover, concavities on the microspheres produced through spray drying are commonly observed due to the rapid evaporation of the solvent. ${ }^{5,15,30}$ However, the high temperature used during spray drying can lead to denaturation of protein carriers $^{5}$ and possibly alter peptide structure due to their reactivity. For instance, non-enzymatic browning can occur if considerable amount of reducing sugar is present in the system. Spray drying technique can be considered more appropriate for micro- rather than nanoencapsulation. Rocha et al. adapted spray drying for encapsulation of peptides for functional food 
application, and also reported to have successfully incorporated the encapsulated product in protein bars. ${ }^{30}$

5.4.3. Coacervation. This technique is considered effective for encapsulation since it is based on electrostatic attraction between the core and wall materials. The technique involves phase separation and deposition of coacervate phase on the core. ${ }^{31}$ Unlike other encapsulation methods, coacervation has been used to achieve EE of up to $91.6 \%$ using similar amounts of core (soy protein and pectin) and wall materials (casein hydrolysate). ${ }^{32}$ The affinity between the core and wall due to surface properties contributed to the resulting high EE. One caveat with achieving such high affinity between the core and wall is that the peptides can be tightly bound to the extent that it becomes difficult to release them when needed. The wall material should have compatible (opposite) charge with the core to be able to coacervate. For instance, anionic polysaccharides such as gum arabic or alginate can be used to coacervate cationic peptides, and vice versa. Another technique used for peptide encapsulation include ionotropic gelation, ${ }^{22}$ which is also a mild preparation method based on electrostatic interactions of the encapsulation materials.

\subsection{Production condition}

The peptide net charge is dependent on the $\mathrm{pH}$ of the medium during encapsulation, and this can influence the EE due to electrostatic effects. Encapsulation with both protein and polysaccharide-based carriers have been found to occur favourably at alkaline $\mathrm{pH} 8 .^{5,15,32}$ Moreover, Ruiz et al. reported that maximum EE was observed at $\mathrm{pH} 10$ with dilute salt $\left(\mathrm{CaCl}_{2}\right)$ solution while the least EE was observed at neutral $\mathrm{pH}$ and high salt concentration. ${ }^{\mathbf{1 6}}$ Conversely, liposome formation has been found to result in higher $\mathrm{EE}$ when conducted at neutral pH. ${ }^{17,24-26}$ Taken together, the size of the encapsulated product is determined by production parameters and inherent properties of the wall and core materials such as energy input per unit mass, surface tension and density.

\section{Release and gastric stability of encapsulated peptides}

High affinity of the core and wall materials is paramount to the formation of stable encapsulated peptide products that can withstand food processing and storage conditions with limited diffusion losses of the core materials. Contrary to EE, a recent study demonstrated that the release kinetics of peptides encapsulated in protein microbeads in aqueous environment was inversely proportional to the peptide hydrophobicity with average release rate constants of 0.1 and $0.014 \mathrm{~min}^{-1}$ for Phe-Trp and LeuTrp-Met-Arg-Phe, respectively, after 1 h. ${ }^{\mathbf{1 4}}$ Conversely, the modification of rapeseed protein by acylation and high pressure treatment that resulted in higher EE was found to increase the \% release of the encapsulated peptide compared to the native protein after $24 \mathrm{~h}$ using the dynamic dialysis method. ${ }^{\mathbf{1 1}}$ This indicates weaker interaction of the peptides with the modified protein carriers. Although theoretically promising, the dearth of experimental information on the biostability of encapsulated protein hydrolysate and peptides makes it difficult to evaluate the prospects of encapsulation in oral delivery of bioactive peptides. A myriad of bioactive peptides derived from various food proteins have been reported and it is becoming increasingly apparent that the focus needs to be shifted to the translation of the peptides into commercial functional food products. Studies focused on characterizing the digestion and release of encapsulated peptides during gastrointestinal processing are crucial in understanding the effect of encapsulation on biostability. One study evaluated the biostability of bioactive peptides encapsulated with a carboxymethylated gum and sodium alginate, and found minimal (up to $10 \%$ ) and maximal (up to $60 \%$ ) release of protein materials after simulated gastric and intestinal digestion phases, respectively. ${ }^{\mathbf{1 6}}$ The released peptides at the intestinal phase can then be presented for absorption into the enterocytes and subsequently into circulation where they are still susceptible to further peptidolytic modification. Therefore, it is imperative to assess the digestion kinetics and biostability of encapsulated peptides, and their bioavailability in different physiological sites to ensure the release of the intact bioactives at appropriate time and target location.

\section{Challenges and future prospects of peptide encapsulation}

The heterogeneity of protein hydrolysates containing diverse range of peptides with different net charge, hydrophobicity, molecular weight and surface properties makes it challenging to achieve high and uniform EE. Enhancing the EE is particularly important to avoid the use of large quantities of the encapsulated protein hydrolysates and peptides in attaining the desired amount of the actual active material. ${ }^{30}$ Purifying the peptides from protein hydrolysates can improve the condition; however, it requires high-end processing techniques that can be uneconomical for small and medium-sized food industry. ${ }^{9}$ However, some techniques are showing promise for use in purifying peptides or concentrating bioactive fractions at a large scale and low cost. ${ }^{334}$ Some promising techniques currently used for the delivery of drug and other bioactive compounds have the potential to be extended to food protein hydrolysates and peptides. For instance, proliposomes, which are used for drug delivery, can be used to overcome the quality issues associated with liposomes such as oxidation, aggregation and phospholipid hydrolysis. ${ }^{35}$

Future studies should focus on detailed and balanced evaluation of encapsulated peptides derived from all the carrier types for biostability, organoleptic properties and bioavailability. The applicable techniques would have to achieve practical EE without requiring expensive processing steps and carrier materials. Forthcoming research should also be focused on understanding the effect of encapsulation on the functionality and stability of encapsulated peptide products, digestion kinetics, release rate, and compatibility with the food and physiological matrices. It is noteworthy that spray dried microspheres have been reported to be highly resistant to mechanical fractures. ${ }^{5}$ Although, peptide release from electrostatically-driven encapsulation (film hydration, 
coacervation) appear relatively easier to achieve, it is imperative to characterize the underlying mechanism and release profile of encapsulated peptide products to facilitate their use in product development. Furthermore, in vivo studies using animal models and human subjects are needed to confirm the effectiveness of encapsulation in enhancing bioavailability and in retention of bioactivity after oral consumption of the products as food.

\section{Conclusion}

Encapsulation of bioactive food compounds is well-positioned to facilitate the design of better and efficient functional foods. This is essential in advancing the research on bioactive food protein hydrolysates and peptides and to develop the market of the peptides as natural health products and nutraceuticals. To achieve high $\mathrm{EE}$, the choice of the carrier material used is dependent on the encapsulation and processing techniques, environment and chemistry of the peptides, although more work is needed to delineate the impact of the latter on EE. Apart from high EE, knowledge of digestion and release kinetics, and the morphology of encapsulated peptide products is paramount to obtaining applicable functional materials for food formulation.

\section{Acknowledgements}

Financial support was provided by the Natural Sciences and Engineering Research Council of Canada (NSERC). A. M. and S. R. C. K. R. are recipients of the Nova Scotia Research and Innovation Graduate Scholarships.

\section{References}

1 C. P. Champagne and P. Frustier, Curr. Opin. Biotechnol., 2007, 18, 184.

2 C. C. Udenigwe, Trends Food Sci. Technol., 2014, 36, 137.

3 M. Fathi, Á. Martin and D. J. McClements, Trends Food Sci. Technol., 2014, 39, 18.

4 D. J. McClements, Nanoparticle-and microparticle-based delivery systems: Encapsulation, protection and release of active compounds, CRC Press, 2014, p. 572.

5 S. E. M. Ortiz, A. Mauri, E. S. Monterrey-Quintero, M. A. Trindade, A. S. Santana and C. S. Favaro-Trindade, LWT-Food Sci. Technol., 2009, 42, 919.

6 G. Picariello, G. Iacomino, G. Mamone, P. Ferranti, O. Fierro, C. Gianfrani, A. Di Luccia and F. Addeo, Food Chem., 2013, 139, 203.

7 M. Segura-Campos, L. Chel-Guerrero, D. A. Betancur-Ancona and V. M. Hernandez-Escalante, Food Rev. Int., 2011, 27, 213.

8 M. Fathi, M. R. Mozafari and M. Mohebbi, Trends Food Sci. Technol., 2012, 23, 13.

9 G. M. Tavares, T. Croguennec, A. F. Carvalho and S. Bouhallab, Trends Food Sci. Technol., 2014, 37, 5.

10 J. J. Ma, X. Y. Mao, Q. Wang, S. Yang, D. Zhang, S. W. Chen and Y. H. Li, LWT-Food Sci. Technol., 2014, 56, 296.

11 Z. Wang, X. Ju, R. He, J. Yuan and L. Wang, Food Bioprocess Technol., 2015, 8, 1305.
12 M. J. Sáiz-Abajo, C. Monzales-Ferrero, A. Moreno-Ruiz, A. Romo- Huald and C. J. Gonzalez-Navarro, Food Chem., 2013, 138, 1581.

13 G. J. O'Neill, T. Egan, J. C. Jacquier, M. O'Sullivan and E. D. O'Riordan, Food Chem., 2014, 160, 46.

14 G. J. O'Neill, T. Egan, J. C. Jacquier, M. O'Sullivan and E. D. O'Riordan, Food Chem., 2015, 180, 150.

15 C. S. Favaro-Trindade, A. S. Santana, E. S. MonterreyQuintero, M. A. Trindade and F. M. Netto, Food Hydrocolloids, 2010, 24, 336.

16 J. C. Ruiz Ruiz, M. R. Segura Campos, D. A. Betancur-Ancona and L. A. Chel-Guerro, ISRN Biotechnol., 2013, 341974, DOI: 10.5402/2013/341974.

17 D. Yokota, M. Moraes and S. C. Pinho, Braz. J. Chem. Eng., 2012, 29, 325.

18 L. E. Kurosawa, K. J. Park and M. D. Hubinger, J. Food Eng., 2009, 94, 326.

19 C. Barbosa, H. A. Morais, F. M. Delvivo, H. S. Mansur, M. C. De Oliveira and M. P. Silvestre, J. Sci. Food Agric., 2004, 84, 1891.

20 J. C. Silva and S. C. Pinho, Int. J. Financ. Stud., 2013, 2, 48.

21 J. A. A. Zhang and J. Pawelchak, Eur. J. Pharm. Biopharm., 2000, 50, 357.

22 B. Zhang and X. Zhang, Biotechnol. Prog., 2014, 29, 1230.

23 S. Yang, X. Y. Mao, F. F. Li, D. Zhang, X. J. Leng, F. Z. Ren and G. X. Teng, Eur. Food Res. Technol., 2012, 235, 91.

24 M. Mosquera, B. Giménez, I. M. da Silva, J. F. Boelter, P. Montero, M. C. Gómez-Guillén and A. Brandelli, Food Chem., 2014, 156, 144.

25 E. da Rosa Zavareze, A. C. Telles, S. L. Mello El Halal, M. da Rocha, R. Colussi, L. M. de Assis, L. A. S. de Castro, A. R. G. Dias and C. Prentice-Hernandez, LWT-Food Sci. Technol., 2014, 59, 841.

26 H. A. Morais, C. M. da Silva Barbosa, F. M. Delvivo, H. S. Mansur, M. C. De Oliviera and M. P. C. Silvestre, J. Food Biochem., 2004, 28, 21.

27 W. Liu, A. Ye, C. Liu, J. Han and H. Singh, Food Chem., 2015, $175,16$.

28 C. M. D. S. Barbosa, H. A. Morais, D. C. F. Lopes, H. S. Mansur, M. C. D. Oliveira and M. P. C. Silvestre, Braz. J. Pharm. Sci., 2002, 38, 361.

29 S. F. Subtil, G. A. Rocha-Selmi, M. Thomazini, M. A. Trindade, F. M. Netto and C. S. Favaro-Trindade, J. Food Sci. Technol., 2014, 51, 2014.

30 G. A. Rocha., M. A. Trindade, F. M. Netto and C. S. FavaroTrindade, Food Sci. Technol. Int., 2009, 15, 407.

31 S. Gouin, Trends Food Sci. Technol., 2004, 15, 330.

32 D. V. Mendanha, S. E. M. Ortiz, C. S. Favaro-Trindade, A. Mauri, E. Monterrey-Quintero and M. Thomazini, Food Res. Int., 2009, 42, 1099.

33 L. Bazinet and L. Firdaous, Recent Pat. Biotechnol., 2013, 7, 9. 34 A. Doyen, C. C. Udenigwe, P. L. Mitchell, A. Marette, R. E. Aluko and L. Bazinet, Food Chem., 2014, 145, 66.

35 B. Zheng, L. Teng, G. Xing, Y. Bi, S. Yang, F. Hao, G. Yan, X. Wang, R. J. Lee, L. Teng and J. Xie, Eur. J. Pharm. Sci., 2015, 77, 254. 